\title{
Una nueva especie de helecho del género Danaea (Marattiales: Marattiaceae) endémica de Costa Rica
}

\author{
Alexander Fco. Rojas Alvarado \\ Jardín Botánico Lankester, Universidad de Costa Rica. Apdo. 1031-7050, Cartago, Costa Rica; afrojasa@yahoo.com
}

Recibido 06-X-2003. C Corregido 08-IX-2005. Aceptado 15-III-2006.

\begin{abstract}
A new species of fern, genus Danaea (Marattiales: Marattiaceae) endemic to Costa Rica. Danaea tuomistoana A. Rojas (Marattiaceae) is described and illustrated as a new species endemic to Costa Rica; it differs from $D$. crispa Endres et Richb. by a truncate blade, and by the pinnae, which are 2/5-1/2 incised, cuneated at the base and with a flat margin. The range of $D$. erecta H. Tuomisto \& R.C. Moran in Costa Rica is expanded with a new locality in Puntarenas (857’15” N-82 50'1” W, 1 500-1 580 masl). Rev. Biol. Trop. 54 (3): 10571060. Epub 2006 Sept. 29.
\end{abstract}

Key words: Danaea, new species, new record, Marattiaceae, ferns, Costa Rica.

Las Marattiaceae son fáciles de reconocer por sus estípulas carnosas y pareadas, pinnas opuestas adheridas al raquis por nudos gruesos y sinangios alongados que se abren por poros o ranuras (Tuomisto y Moran 2001).

El género Danaea se reconoce por estípulas en la base del pecíolo, hojas usualmente 1-pinnadas con pinnas opuestas y nudos gruesos en la unión de las pinnnas con el raquis. Danaea es enteramente neotropical con alrededor de 40 especies descritas; sin embargo, es un grupo que requiere revisión debido al alto grado de sinonimia aún desconocida (Tuomisto y Moran 2001).

\section{MATERIALES Y MÉTODOS}

La nueva especie ha sido así considerada después de comparar con especies afines en distintos tratamientos taxonómicos para México, Centroamérica y Sudamérica (Smith 1981, Stolze 1983, Mickel y Beitel 1988, Tryon y Stolze 1989, Lellinger 1989, Camus y Pérez 1995, y Tuomisto y Moran 2001), y revisar las colecciones de: Museo Nacional de Costa Rica (CR) e Instituto Nacional de Biodiversidad (INB).

\section{RESULTADOS}

\section{Danaea tuomistoana}

A. Rojas, sp. nov.

Tipo: COSTARICA. Puntarenas: Cantón de Golfito, Parque Nacional Corcovado, Península de Osa, Cerro Rincón, 8³1’ N, 83²8’ W, 700745 msnm, A. Rojas et al. 4217 (Holotipo: INB, 35513; Isotipos: CR, MO) (Fig. 1).

Diagnósis latina: D. crispa Endres et Richb. f. affinis, sed laminae truncata, pinnis basalis cuneatis majoribus incisus et absque crispatis differt.

English diagnosis: The new species differs from D. crispa Endres et Richb. by a truncate blade, and a more incised pinna with cuneate base and flat margin. 


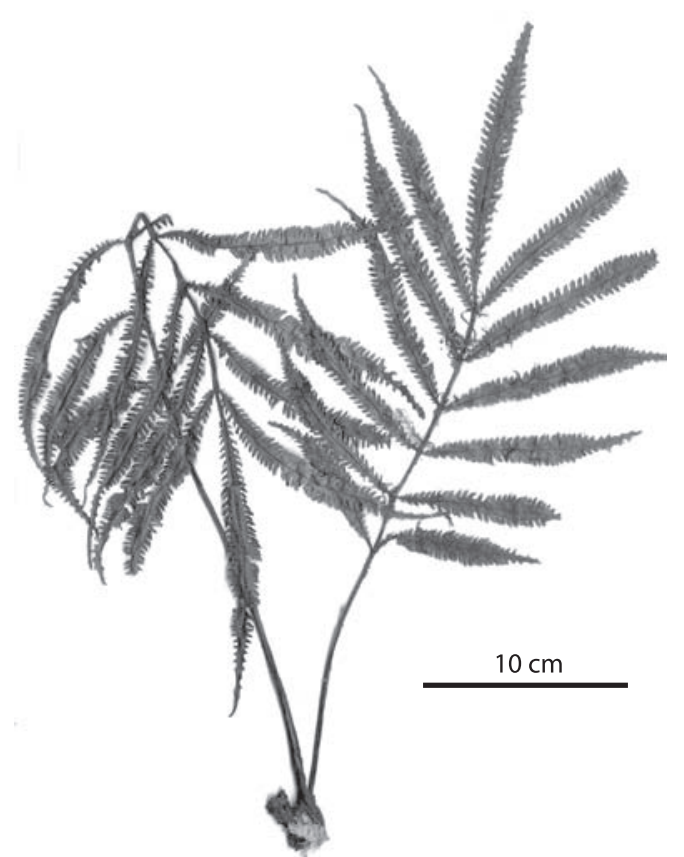

Fig. 1. Ejemplar tipo de D. tuomistoana (A. Rojas et al. 4217, CR) (escala: $10 \mathrm{~cm}$ ).

Fig. 1. Type specimen of D. tuomistoana (A. Rojas et al. 4217, CR) (scale: $10 \mathrm{~cm}$ ).

Descripción: Rizoma de 5-12 mm de diámetro (incluyendo las aurículas), corto reptante a ascendente; hojas de $44-58 \mathrm{~cm}$ de largo, próximas; pecíolo de 18-24 cm de largo, aprox. 2/5 del tamaño de la hoja, pardo, sin entrenudos; escamas de la base del pecíolo de $1-3 \times 0.5-1.0 \mathrm{~mm}$, ovadas a lanceoladas, pardas, basifijas, enteras; escamas del resto del pecíolo y raquis de 0.2-0.4 $\mathrm{mm}$ de diámetro, redondas a ovadas, pardas, peltadas, enteras; lámina 1-pinnado-pinnatífida, ovada a ovadolanceolada, con 7-9 pares de pinnas, base truncada, ápice agudo, haz y envés del mismo color, paripinnada o inparipinnada, sin yemas terminales; raquis alado en $1 / 2-2 / 3$ de la parte distal de la lámina, el ala hasta de $1.5 \mathrm{~mm}$ de ancho, más ancha debajo de la unión de las pinnas y ausente inmediatamente después de la unión de estas; pinnas de 8-14x1.3-1.8 cm, oblanceoladas, opuestas, incisas 2/5-1/2 de la distancia entre el margen y la costa y con el lóbulo basal basiscópico libre, base cuneada,

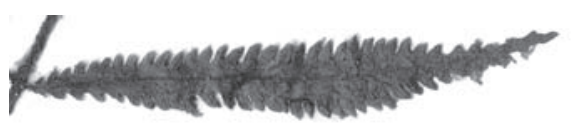

$10 \mathrm{~cm}$

Fig. 2. Pinna media de D. tuomistoana mostrando los detalles (escala: $10 \mathrm{~cm}$ ).

Fig. 2. Middle pinna of $D$. tuomistoana with details (scale: $10 \mathrm{~cm})$.

ápice largamente acuminado; escamas de las costas similares a las del raquis pero un poco onduladas; escamas del envés de 0.1-0.3 mm de diámetro, redondas, onduladas; escamas de la haz de las costas y tejido laminar de 0.3$1 \mathrm{~mm}$, redondas a ovado-lanceoladas, pardas, margen dentado a largo ciliado; venas simples a raramente bifurcadas, una por lóbulo; hojas fértiles no vistas.

Hábitat: Presente en zonas poco iluminadas a orillas de riachuelos entre los 700-745 msnm.

Distribución: Hasta ahora sólo se ha recolectado en el Cerro Rincón, Península de Osa, Costa Rica.

Etimología: Esta especie es dedicada a Hanna Tuomisto por sus importantes aportes al género Danaea.

\section{Nuevo registro}

Danaea erecta H. Tuomisto et R.C. Moran, Fl. Ecuador 66: 37-40. 2001. Tipo: Ecuador, Pichincha, Maquipucuna Biological Field Station, ca. $5 \mathrm{~km}$ E of Nanegalito, $0^{\circ} 7^{\prime}$ N, 78³8' W, 1 400-1 700 msnm, R. Moran et al. 5950 (Holotipo: QCA; Isotipos: AAU, NY, QCNE, TUR, UC).

Distribución general: Costa Rica, Colombia y Ecuador.

Material de nueva distribución: Costa Rica. Puntarenas: Cantón de Coto Brus, Zona Protectora Las Tablas, Cordillera de Talamanca, Sabalito, Las Alturas de Cotón, 
Estación Biológica Las Alturas, 857’15” N8250’1” W, 1 500-1 580 msnm, A. Rojas 899 (INB, CR) (Fig. 3).

Comentario: En Costa Rica esta especie se diferencia de $D$. nodosa (L.) Sm. por rizoma ascendente (vs. rastrero), pinnas $4-5 \mathrm{~cm}$ de ancho (vs. 2.0-3.5 cm), oblanceladas (vs. angostamente oblongas), pinnas basales

8-10 cm distantes entre sí (vs. 5-7 cm distantes), base de las pinnas angostamente cuneada (vs. anchamente cuneada a obtusa) y con unión atenuada (vs. unión abrupta); además de estar distribuida a mayor elevación [1 500-1 580 msnm vs. 0-800 (-1 200) msnm].

Se diferencia del material sudamericano por pinnas oblanceoladas (vs. oblongas) y pinnas basales con ápice aristado (vs. cuspidado), por lo demás no parece presentar diferencias significativas. Por no presentar yema apical se parece al material del lado este de los Andes indicado por Tuomisto y Moran (2001).

\section{DISCUSIÓN}

D. tuomistoana se parece a Danaea crispa Endres et Richb. f. por presentar pinnas

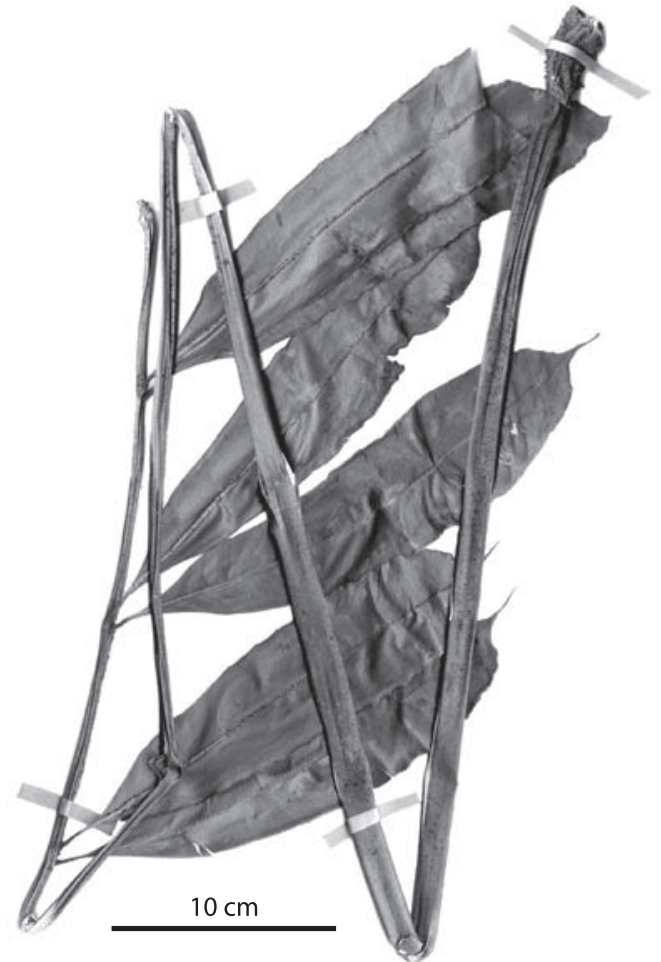

Fig. 3. Ejemplar del nuevo registro de D. erecta $\mathrm{H}$. Tuomisto et R.C. Moran (A. Rojas 899, INB) (escala: $10 \mathrm{~cm}$ ).

Fig. 3. Representative specimen of the new record $D$. erecta H. Tuomisto et R.C. Moran (A. Rojas 899, INB) (scale: $10 \mathrm{~cm}$ ).

\section{CUADRO 1}

Diferencias morfológicas entre la nueva especie y las especies de Danaea más relacionadas con D. tuomistoana (D. crispa $y$ D. moritziana)

\section{TABLE 1}

Morphological difference between the new species and the Danaea species more related to D. tuomistoana (D. crispa and D. moritziana)
D. tuomistoana
D. crispa
D. moritziana

Tamaño de las hojas

Disposición de las hojas

Base de la lámina

Base de las pinnas

Ápice de las pinnas

Margen de las pinnas

Yema apical

Venas

Color del envés de la lámina
44-58 cm de largo

Poco inclinadas

Truncada

Cuneada

Acuminado

Inciso 2/5-1/2, plano

Ausente

Comúnmente simples

Verde
20-28 cm de largo

Muy inclinadas

Cuneada

Truncada

Obtuso a redondo

Inciso 1/6-1/4, crispado

Presente

Simples o bifurcadas en la base

Verde
60-120 cm de largo

Poco inclinadas

Truncada

Obtusa a redonda

Acuminado

Entero, plano

Presente o ausente

Comúnmente simples

Glauco 
incisas con un lóbulo por vena y por el raquis alado, pero se diferencia por hojas más grandes (44-58 cm de largo vs. $20-28 \mathrm{~cm}$ ) y poco inclinadas (vs. muy inclinadas), lámina con la base truncada (vs. cuneada) y sin yema apical (vs. con yema), pinnas con la base cuneada (vs. truncada), con el ápice acuminado (vs. obtuso a redondo) e incisiones más profundas (2/5-1/2 de la distancia entre el margen y la costa vs. 1/7-1/4) y venas casi siempre simples (vs. simples o bifurcadas) (Fig. 2) (Cuadro 1).

Se parece también a $D$. moritziana C. Presl por la base de la lámina truncada y las pinnas con ápice acuminado a cuspidado, pero difiere por lámina con color homogéneo en ambas superficies (vs. glauca en el envés), pinnas incisas (vs. enteras) y con la base cuneada (vs. obtusa a redonda) (Cuadro 1).

En este trabajo se usa a $D$. moritziana como especie válida y diferente de $D$. cuspidata Liebm., por nervaduras comúnmente no bifurcadas en la base (vs. bifurcadas) y ligeramente más oscuras que la lámina (vs. más oscuras que la lámina) (Lellinger 1989). Adicionalmente, la lámina de $D$. moritziana tiene el envés glauco (vs. ambas superficies verdes en $D$. cuspidata) y el raquis es alado en toda su extensión o al menos en los 2/3 terminales (vs. exalado o alado sólo apicalmente en $D$. cuspidata).

\section{AGRADECIMIENTOS}

Agradezco el apoyo de los herbarios consultados por permitirme utilizar sus colecciones. Un reconocimiento especial a los revisores anónimos, por sus comentarios y recomendaciones hechos al manuscrito. Agradezco también a Armando Ruiz (CR) y a Alejandro
Herrera (INB) por ayudarme a obtener las imágenes utilizadas en este artículo.

\section{RESUMEN}

Se describe e ilustra una especie nueva endémica de helecho de Costa Rica, Danaea tuomistoana A. Rojas (Marattiaceae), caracterizada porque las pinnas son incisas 2/5-1/2 entre la costa y el margen, y son cuneadas en la base. Se amplia el ámbito geográfico de $D$. erecta $\mathrm{H}$. Tuomisto \& R.C. Moran a Puntarenas, Costa Rica.

Palabras clave: Danaea, nueva especie, nuevo registro, Marattiaceae, helechos, Costa Rica.

\section{REFERENCIAS}

Camus, J.M. \& B. Pérez. 1995. Danaea, p. 48-50. In R.C. Moran \& R. Riba (eds.). Flora Mesoamericana. Vol. 1. Psilotaceae a Salviniaceae. Universidad Nacional Autónoma de México, D.F., México.

Lellinger, D.B. 1989. The ferns and fern-allies of Costa Rica, Panama, and the Chocó. Part I: Psilotaceae through Dicksoniaceae. Pteridología 2A: 85-90.

Mickel, J.T. \& J.M. Beitel. 1988. Pteridophyte Flora of Oaxaca, Mexico. Mem. New York Bot. Gard. 46: 144-145.

Smith, A.R. 1981. Pteridophytes, p. 88-89. In D.E. Breedlove (ed.). Flora of Chiapas 2. Calif. Acad. Sci., San Francisco, EEUU.

Stolze, R.G. 1983. Ferns and fern allies of Guatemala. Part III. Psilotaceae through Isoetaceae. Fieldiana, Bot, n.s. 12 : 1-91.

Tryon, R.M. \& R.G. Stolze. 1989. Pteridophyta of Peru. Part I. 1. Ophioglossaceae -12. Cyatheaceae. Fieldiana, Botany n.s. 20: 15-20.

Tuomisto, H. \& R.C. Moran. 2001. Marattiaceae, p. 21-68. In G. Harling \& L. Andersson (eds.). Flora of Ecuador. Nº6. 6. Ophioglossaceae-10. Gleicheniaceae. Copenague, Dinamarca. 